\title{
Musique et langage chez Rousseau, études présentées par Claude Dauphin
}

\section{Paola Sosso}

\section{(2) OpenEdition}

10 Journals

\section{Édition électronique}

URL : http://journals.openedition.org/studifrancesi/33287

DOI : 10.4000/studifrancesi.33287

ISSN : 2421-5856

Éditeur

Rosenberg \& Sellier

\section{Édition imprimée}

Date de publication : 1 décembre 2005

Pagination : $649-650$

ISSN : 0039-2944

\section{Référence électronique}

Paola Sosso, « Musique et langage chez Rousseau, études présentées par Claude Dauphin », Studi

Francesi [En ligne], 147 (XLX | III) | 2005, mis en ligne le 30 novembre 2015, consulté le 18 avril 2021.

URL : http://journals.openedition.org/studifrancesi/33287 ; DOI : https://doi.org/10.4000/

studifrancesi.33287

Ce document a été généré automatiquement le 18 avril 2021.

\section{(c)}

Studi Francesi è distribuita con Licenza Creative Commons Attribuzione - Non commerciale - Non opere derivate 4.0 Internazionale. 


\title{
Musique et langage chez Rousseau, études présentées par Claude Dauphin
}

\author{
Paola Sosso
}

\section{RÉFÉRENCE}

Musique et langage chez Rousseau, études présentées par Claude DAUPHIN, Oxford, Voltaire Foundation, («SVEC 2004», 8), 2004, pp. 261.

1 Ce volume regroupe les études présentées lors du XII colloque de l'Association Rousseau qui a eu lieu du 24 au 27 mai 2001 au Département de musique de l'Université du Québec à Montréal. Musique et langage, thèmes centraux des interventions, représentent les deux pôles de la pensée de Rousseau, qui souligne à plusieurs reprises l'exclusivité humaine de la parole et de la musique (Essai sur l'origine des langues, Dictionnaire de musique). Les relations équivoques entretenues par ces deux termes sont symboliquement évoquées dans Pygmalion, nous suggère Claude Dupin dans Le vertige des origines (pp. ix-xiv), brève introduction au volume qui situe les contributions à ce collectif dans la perspective des études rousseauistes modernes. La première section, "L'espace des voix», s'ouvre avec un article de Catherine KINTZLER (Musique, voix, intériorité et subjectivité: Rousseau et les paradoxes de l'espace, pp. 3-19) qui montre de quelle manière la mise en place du système vocalique chez l'homme primitif marque le moment de la formation de l'idée d'autrui; la pensée de l'identification et de l'altérité se traduit en notions de proximité et d'éloignement, d'intérieur et d'extérieur, tramant ainsi l'histoire de la subjectivité. L'intervention qui suit, de Jean-François PERRIN, s'occupe de La musique dans les lettres selon Rousseau: une écoute du sensible (pp. 20-29): l'auteur se concentre sur le rapport musique/parole à partir de quelques éléments de la pensée théorique de Rousseau et en tenant compte des caractéristiques peu musicales de la langue française. Martin STERN, dans Le problème de la conversion dans la pensée 
musicale de Rousseau (pp. 30-38) s'arrète sur un volte-face qui est déterminant pour la réflexion esthétique rousseauiste: d'abord partisan du goût musical français, JeanJacques devient en effet, à partir de 1744, un fervent admirateur du style italien. La dernière contribution de cette section, de Jean FISETTE, propose enfin une approche sémiotique de l'Essai sur les origines des langues (La genèse du sens chez Rousseau, pp. 39-47). «La musique éloquente» est le thème de la deuxième partie, où l'étude de John T. ScotT (Climate, causation and the power of music in Montesquieu and Rousseau, pp. 51-61) analyse les éléments climatiques, historiques, et géographiques de l'Essai sur l'origine des langues montrant de quelle manière Roussau reprend et développe la théorie contenue dans l'Esprit des lois sur l'influence du climat. Mira MOGERNSTERN, dans Jean-Jacques Rousseau: music, language and politics (pp. 62-74), dirige son attention sur le rapport entre le langage et la politique, mettant en lumière la fonction musicale du langage et le rôle central de la parole dans la formation de la polis. L'approche originale d'Alexandra cooK parvient à mettre en lumière une même destinée taxinomique sur laquelle se greffent les systemes de notation de la musique et de la botonique (Rousseau and the languages of music and botany, pp. 75-87). On revient au rapport langage-politique avec l'intervention d'Ourida MostefaI, Inventer un langage nouveau: Rousseau et la polémique (pp. 88-92), qui part du discours de Fabricius dans le Discours sur les sciences et les arts pour montrer que ce langage, radicalement nouveau dans son renversement de l'usage social établi, trouve de nombreux échos dans toutes les œuvres du genevois. Dans l'essai qui clôt la section, L'Arménien de Venise: validation sémiologique ou ethnomusicologie (pp. 93-99), Danick TROTTIER nous propose une perspective sémiotique pour l'analyse de cet épisode. La troisième partie a pour titre «Entre silence et désir»: la grille psychanalytique, à laquelle recourent Michel sнмочснкоуттсн ( $L a$ Fonction du désir dans l'origine des langues selon Rousseau, pp. 103-111), Catherine J. COLE (From silence to society: the conflicting musical visions of Rousseau's «Discours sur l'origine de l'inégalité» and "Essai sur l'origine des langues», pp. 112-121) et Jeff BLACK (The dupes of words: the problem and promise of language in Rousseau '"Discours sur l'origine de l'inégalité», pp. 122-130) nous aide à percevoir combien le désir narcissique de l'autre soi-mème, la relation entre individu et société, le rapport entre passion et compassion se réalisent, dans les textes de Rousseau, grâce à la séduction du langage et de la musique. Michel TERMOLLE (L'Éducation négative dans l'apprentissage de la musique, pp. 131-143) part d'une intéressante relecture de l'Emile, de la Dissertation sur la musique moderne et du Projet concernant de nouveaux signes pour la musique pour nous amener à découvrir les principes pédagogiques de l'éducation négative dans l'apprentissage de la musique. Jean-Paul DESPINS, auteur du dernier essai de la troisième partie, nous propose finalement une analyse consacrée à La dichotomie rousseauiste langue et musique, revue par la biomusicologie et la neuromusicologie (pp. 144-151). On arrive ainsi à la quatrième section, "De la culture et du politique», contenant quelques relectures critiques de la pensée musicale de Rousseau sous l'éclairage des sciences politiques: telles sont les études de José Oscar DE ALMEDA MARQUES (The politics of taste: a piace for art music in Rousseau's construction of the political community, pp. 155-164), celle de Stuart A. MACNIVEN (Politics, language and music in the unity of Roussau's system, pp. 165-174), celle de Christopher BERTRAM (Language, music and the transparent society in Rousseau's "Essai sur l'origine des langues» and the "Contrat social», pp. 175-182) et celle de Julia sIMON (Music and the performance of community in Rousseau, pp. 192-200). La contribution de Melissa A. BUTLER tient au contraire au rapport entre musique et psychosociologie (The quarrel between Kiiuiseau and Rameau: evidence from 
contemporary psychology, pp. 183-191): l'auteur nous conduit à travers les étapes de la célèbre querelle entre Rousseau et Rameau relue à la lumière des connaissances de la psychologie contemporaine. La dernière section, «L'opéra comme représentation», s'occupe de la tribune qui est propre à la musique, l'opéra. Pierre SABYN (Accent, expression, unité de mélodie dans le «Devin du village»: la théorie esthétique éclairée par l'analyse musicale, pp. 203-210) nous présente Le divin du village comme lieu d'essai de mise en œuvre de conceptions et de principes inconnus des Français, à savoir la représentation du peuple à l'opéra. Pamela GAY-WHITE (Rousseau and the lyric natural: the representation of «Le divin du village», pp. 211-219), se penche encore une fois sur l'analyse du même texte, tandis que la perspective s'ouvre à une mise en rapport avec Berlioz dans l'intervention de Guillaume BORBRY, Hector juge de Jean-Jacques: Berlioz lecteur et auditeur de Rousseau (pp. 220-228): Berlioz cite Rousseau au nom d'un aspect précis, son double statut d'homme de lettres et de compositeur, et il est alors intéressant de suivre, sur le chemin tracé par l'auteur, la reprise et l'utilisation chez Berlioz des œuvres du genevois. Essayer de comprendre par quelles voies Rousseau est devenu l'auteur de la Lettre sur la musique française, comprendre l'impact qu'a eu sur lui La lettre sur Omphale de Grimm, tel est le but de l'étude de Jacqueline WAEBER (Paysage d'avant querelle: Rousseau continuateur de Grimm, pp. 229-249) qui clôt le voleparcourant les années de formation de Rousseau, présage des querelles à venir. 